\title{
Does Cryopreservation of Ovarian Tissue Affect the Distribution and Function of Germinal Vesicle Oocytes Mitochondria?
}

\author{
Mojdeh Salehnia, ${ }^{1}$ Virpi Töhönen, ${ }^{2}$ Saeed Zavareh, ${ }^{3}$ and Jose Inzunza ${ }^{2}$ \\ ${ }^{1}$ Anatomy Department, Tarbiat Modares University, P.O. Box 14115-111, Tehran, Iran \\ ${ }^{2}$ Division of Medical Nutrition, Department of Biosciences and Nutrition, Karolinska University Hospital, Karolinska Institutet, \\ Novum, 14186 Stockholm, Sweden \\ ${ }^{3}$ School of Biology, Damghan University, P.O. Box 3671641167, Damghan, Iran
}

Correspondence should be addressed to Mojdeh Salehnia; salehnim@modares.ac.ir

Received 6 April 2013; Revised 16 June 2013; Accepted 17 June 2013

Academic Editor: Heide Schatten

Copyright (C) 2013 Mojdeh Salehnia et al. This is an open access article distributed under the Creative Commons Attribution License, which permits unrestricted use, distribution, and reproduction in any medium, provided the original work is properly cited.

\begin{abstract}
The aim of this study was to evaluate mitochondrial alteration and ATP content of germinal vesicle (GV) oocytes isolated from fresh and vitrified ovaries. After superovulation, the ovaries from adult mice were collected and divided into control and vitrified groups. GV oocytes were isolated mechanically from each group. Half were cultured for 24 hours and their maturation was assessed. Metaphase II oocytes were collected and submitted to in vitro fertilization and their fertilization rates and development to the blastocyst stage were evaluated. In the remaining GV oocytes, ATP levels were quantified, and mitochondrial distribution, mitochondrial membrane potential, and intracellular free calcium were detected with rhodamine 123, JC-1 and Flou-4 AM staining, using laser-scanning confocal microscopy. Maturation and fertilization rates of GV oocytes and the developmental rates of subsequent embryos were significantly lower in vitrified samples $(P<0.05)$. The ATP content and $\mathrm{Ca}^{2+}$ levels differed significantly in fresh and vitrified GV oocytes $(P<0.05)$. Most mitochondria were seen as large and homogenous aggregates $(66.6 \%)$ in fresh GV oocytes compared to vitrified oocytes (50\%). No significant differences in mitochondrial membrane potential were found between the groups. The lower maturation and fertilization rates of GV oocytes from vitrified ovaries may be due to changes in their mitochondrial function and distribution.
\end{abstract}

\section{Introduction}

Vitrification is a method for cryopreservation of oocytes, embryos, and ovarian tissue. The survival and development rates of vitrified oocytes, embryo, and follicles have been studied [1-4]. The vitrified oocytes and follicles have been found to mature somewhat more slowly than fresh samples [2-6]. However, the further development of vitrified follicles compared with nonvitrified samples is controversial [2, 3, 6-8].

Cryopreservation causes alterations in the physical and chemical properties of oocytes and embryos, including loss of cell membrane and cytoskeletal integrity, mitochondrial depolarization, and increased production of reactive oxygen species [9-15]. These alterations are associated with osmotic forces created during dehydration, cooling, rehydration, and warming and may affect mainly cytoplasmic activities such as mitochondrial function, metabolism, and intracellular signaling pathways.

The number and distribution of mitochondria and energy (ATP) production are critical factors that influence not only the maturation and development of the oocyte but also its fertilization and subsequent embryo development [16-20]. Structural and metabolic mitochondrial defects are associated with failures in oocyte maturation and abnormal development or arrest of embryos [21-24]. Reports on vitrification methods using different cryoprotectants show that mitochondrial distribution is affected in mouse metaphase II (MII) oocytes and embryo at two pronucleus stages [11, $12,15]$. However, impairment of mitochondrial function by vitrification was shown in bovine and human oocytes $[14,25]$. Recently, Demant et al. showed transient changes in mouse oocyte mitochondrial activity after vitrification [11]. 
In vitro maturation of isolated follicles and germinal vesicle $(\mathrm{GV})$ oocytes from vitrified ovarian tissue may be an alternative technique for preserving fertility potential in parallel with oocyte preservation. Several previous reports were focused on vitrification of single oocytes or follicles, then their development was assessed [1-4] whereas, to our knowledge, no research has documented the effects of ovarian vitrification on the maturation and distribution of mitochondria and ATP content of isolated GV oocytes.

Dysfunction of oocyte mitochondria may occur without detectable morphological abnormalities. Therefore, we studied the effects of ovarian vitrification on mitochondrial distribution and function in mouse GV oocytes using mitochondria-specific fluorescent probes. The energy (ATP) and free $\mathrm{Ca}^{2+}$ concentrations and the developmental potential of the GV oocytes were also evaluated.

\section{Materials and Methods}

All chemicals were purchased from Sigma-Aldrich (St. Louis, USA), unless otherwise noted.

2.1. Animals and Collection of Ovarian Tissue. Adult female FVB/N mice, 6-8 weeks' old, were housed under controlled conditions (12 h light/12 h dark) with free access to water and food. The mice were superovulated using an i.p. injection of $10 \mathrm{IU}$ pregnant mare serum gonadotropin. After $48 \mathrm{~h}$, superovulated female mice were sacrificed by cervical dislocation and ovaries were dissected, freed of fat and mesentery, and divided into control (nonvitrified) and vitrified groups. Animal experiments were performed at the infection-free animal facility of Karolinska University Hospital in accordance with the ethical committee's approval.

2.2. Vitrification and Warming. The vitrification procedure was based on a method used earlier [26]. Briefly, ovarian tissue was equilibrated in a vitrification medium (EFS40) containing 40\% ethylene glycol (V/V), 30\% Ficoll 70 (W/V), and $1 \mathrm{M}$ sucrose supplemented with bovine serum albumin (BSA) for 5 minutes at room temperature. The ovaries were placed in plastic cryotubes with a minimum volume of vitrification medium $(10 \mu \mathrm{L})$, then put on nitrogen vapor for 20 seconds, immersed in liquid nitrogen, and maintained for 1 week. Vitrified ovaries were warmed at room temperature and placed in a $25^{\circ} \mathrm{C}$ water bath for 20 seconds. The contents of each cryotube were placed in $1 \mathrm{~mL}$ of descending concentrations of sucrose $(1$ and $0.5 \mathrm{M})$ at room temperature for 5 minutes. Warmed ovaries were equilibrated for 30 minutes in tissue culture medium (TCM 199; Gibco, Grand Island, NY, USA) supplemented with $5 \mathrm{mg} / \mathrm{mL}$ BSA before isolation and collection of GV oocytes.

2.3. Isolation and Collection of the Germinal Vesicle Oocytes. The GV oocytes ( $n=441$ for fresh and 328 for vitrified groups) were mechanically isolated from large preantral follicles from fresh and vitrified-thawed ovaries using 29 gauge needles during five minutes. Oocytes with a prominent GV and clear ooplasm (average $90 \mu$ diameter) were selected for observation using a stereomicroscope. The survival rate of isolated oocytes was assessed morphologically. Collected oocytes were used for the following experiments.

2.4. In Vitro Maturation of GV Oocytes. The maturation medium TCM199 was supplemented with $50 \mu \mathrm{g} / \mathrm{mL}$ penicillin, $75 \mu \mathrm{g} / \mathrm{mL}$ streptomycin, $0.23 \mathrm{mM}$ sodium pyruvate, $10 \%$ fetal bovine serum, $75 \mathrm{mIU} / \mathrm{mL} \mathrm{rFSH}$, and $10 \mathrm{IU} / \mathrm{mL}$ hCG (Serono, Switzerland). GV oocytes ( $n=261$ for control and 183 for vitrified group) were cultured in $30 \mu \mathrm{L}$ drops of maturation medium under mineral oil at $37^{\circ} \mathrm{C}, 100 \%$ humidity in $5 \%$ $\mathrm{CO}_{2}$ for $18 \mathrm{~h}$. At the end of the culture period, the number of oocytes at the GV stage, germinal vesicle breakdown (GVBD), and MII was counted using an inverted microscope. MII oocytes were collected and used for in vitro fertilization.

2.5. In Vitro Fertilization and Embryo Culture. Cauda epididymes were dissected from mature male mice and placed into $500 \mu \mathrm{L}$ of T6 medium with $5 \mathrm{mg} / \mathrm{mL}$ BSA under mineral oil drops containing freshly released spermatozoa were placed in $37^{\circ} \mathrm{C}$ and $5 \% \mathrm{CO}_{2}$ incubator for capacitation.

The collected MII oocytes from control $(n=226)$ and vitrified $(n=92)$ groups were transferred to T6 medium supplemented with $15 \mathrm{mg} / \mathrm{mL}$ BSA and containing capacitated spermatozoa. 4-6 hours later, the oocytes were transferred to $10 \mu \mathrm{L}$ drops of T6 medium with $5 \mathrm{mg} / \mathrm{mL}$ BSA. The presence of 2 pronucleus zygote was considered as successful fertilization. Embryos were observed daily under an inverted microscope, and the number of embryos reaching 2-cell, 4-cell, morula, and blastocyst stages was recorded for 120 hours [27].

2.6. Measurement of Cytoplasmic ATP Content. The ATP content of oocytes was measured using methods described previously [28]. Briefly, GV stage oocytes were isolated from control $(n=30)$ and vitrified samples $(n=25)$ and rapidly frozen individually in microtubes containing $200 \mu \mathrm{L}$ of ultrapure water at $-80^{\circ} \mathrm{C}$. ATP level of each single oocyte was quantified by measuring luminescence (Berthold LB 9501 luminometer) in an ATP-dependent luciferin-luciferase bioluminescence assay (Bioluminescence Somatic Cell Assay System; Sigma, USA).

A standard curve with different ATP concentrations was generated for each series of analyses. ATP content was determined by the standard curve.

2.7. Visualization of Mitochondria Using Rhodamine 123 (R123). Viable mitochondria were identified using R123 (Molecular Probes, Invitrogen, Eugene, OR, USA) staining as described by Van Blerkom et al. [29]. A stock solution of $10 \mu \mathrm{g} / \mathrm{mL}$ R123 in dimethylsulfoxide was prepared and stored at $-20^{\circ} \mathrm{C}$. GV oocytes from both study groups (at least 20 oocytes per each group) were stained with $1 \mu \mathrm{g} / \mathrm{mL}$ R123 in TCM medium for $10 \mathrm{~min}$ in the dark at $37^{\circ} \mathrm{C}$. After washing samples in TCM medium, oocytes were visualized using LSCM (Olympus, Segrate, Italy) to detect and capture serial sections of oocytes for assessment of cytoplasmic distribution of R123 (520 nm emission).

2.8. Intracellular Free Calcium Analysis by Fluo-4 AM. The free $\mathrm{Ca}^{2+}$ content of the oocytes was detected by LSCM as 
TABLE 1: The maturation rates of the mouse vitrified and nonvitrified tissue-isolated GV oocytes.

\begin{tabular}{lcccccccc}
\hline Groups & $\begin{array}{c}\text { No. of } \\
\text { isolated } \\
\text { GV }\end{array}$ & $\begin{array}{c}\text { No. of GV } \\
\text { reached MII }\end{array}$ & $\begin{array}{c}\text { No. of } \\
\text { degenerated }\end{array}$ & $\begin{array}{c}\text { No. of } \\
\text { fertilized }\end{array}$ & No. of 2 cell & No. of 8 cell & No. of morula & $\begin{array}{c}\text { No. of } \\
\text { blastocyst }\end{array}$ \\
\hline Control & 261 & 226 & 6 & 183 & 163 & 100 & 86 & 75 \\
& & $(86.9 \pm 4.51)$ & $(2.19 \pm 1.01)$ & $(81 \pm 3.46)$ & $(89 \pm 4.24)$ & $(54.6 \pm 3.79)$ & $(46.9 \pm 3.82)$ & $(40.9 \pm 2.74)$ \\
Vitrification & 183 & $92^{*}$ & $30^{*}$ & $44^{*}$ & $28^{*}$ & $12^{*}$ & $7^{*}$ & $5^{*}$ \\
\end{tabular}

Four experimental replicates were performed for each group. GV: germinal vesicle stage oocyte; MII: metaphase II oocyte. Data within prentices are \% \pm SD.

* Significant differences compared to the control group in the same column $(P<0.05)$.

average relative fluorescence intensity $(\mathrm{RFI})$ in individual fresh $(n=20)$ and vitrified GV oocytes $(n=25)$ according to Jones et al.s [9] protocol. Briefly, oocytes were preloaded for $60 \mathrm{~min}$ in TCM medium supplemented with $4 \%$ BSA and $20 \mu \mathrm{mol} / \mathrm{L}$ Fluo- $4 \mathrm{AM}$ (Molecular Probes, Invitrogen, Eugene, OR, USA), followed by a $30 \mathrm{~min}$ wash in normal medium. Oocytes were transferred to an LSCM chamber maintained at $37^{\circ} \mathrm{C}$. For quantifying relative RFI, a long path filter with an emission detection $>510 \mathrm{~nm}$ was used and digital images were processed.

2.9. Determination of Mitochondrial Membrane Potential $(\triangle \Psi)$ by JC-1. Mitochondrial potential assays were performed according to the method of Smiley et al. [30]. The potential sensitive fluorescence dye JC-1 $\left(5,5^{\prime} 6,6^{\prime}\right.$-tetrachloro-1,1,3,3' -tetraethylbenzimidazolycarbocyanine iodide (Molecular Probes, Invitrogen, Eugene, OR, USA) was used to measure the activity of oocyte mitochondria.

JC-1 dye was dissolved in dimethylsulfoxide at $10 \mu \mathrm{g} / \mathrm{mL}$ as a stock solution and diluted into preequilibrated TCM medium (Sigma) at $1 \mu \mathrm{g} / \mathrm{mL}$, using a vortex. GV oocytes were isolated from fresh $(n=20 \mathrm{GV})$ and vitrified $(n=$ $15 \mathrm{GV}$ ) ovaries, exposed to the dye at $37^{\circ} \mathrm{C}$ in a $5 \% \mathrm{CO}_{2}$ incubator for $10 \mathrm{~min}$ in TCM medium, and then washed in TCM medium. The relative fluorescence intensity of JC-1 stained oocytes was determined by LSCM. The distribution of JC-1 monomer (green fluorescence detected in the fluorescein isothiocyanate channel, $515-530 \mathrm{~nm}$ bandpass filter) and Jaggregate fluorescence (orange/red J-aggregate fluorescence) was detected in the rhodamine isothiocyanate channel, using $585 \mathrm{~nm}$ long path filter. Laser power and photomultiplier settings were kept constant for all experiments. Images of individual zygotes were captured and quantified for fluorescence intensity.

2.10. Detection of ROS Content in GV Oocytes Using Spectrofluorometer. To measure ROS levels in GV oocytes derived from vitrified $(n=100)$ and nonvitrified $(n=100)$ mouse ovaries, after mechanical isolation of oocytes from ovaries, 20 oocytes were pooled in each experiment $(n=60$ in five repeats in each group), washed 3 times with phosphate buffer saline (PBS), and incubated in $40 \mathrm{mmol} / \mathrm{L}$ of Tris- $\mathrm{HCl}$ buffer $(\mathrm{pH}=7.0)$ containing $5 \mu \mathrm{mol} / \mathrm{L} 2^{\prime}, 7^{\prime}$-dichlorodihydrofluorescein diacetate (DCHFDA; Merck; Darmstadt, Germany) at $37^{\circ} \mathrm{C}$ for $30 \mathrm{~min}$. Then, the solution was removed and the oocytes were washed three times with PBS, immediately homogenized in $100 \mu \mathrm{L}$ of Tris- $\mathrm{HCl}$ buffer $(40 \mathrm{mmol} / \mathrm{L}, \mathrm{pH} 7.0)$, sonicated at

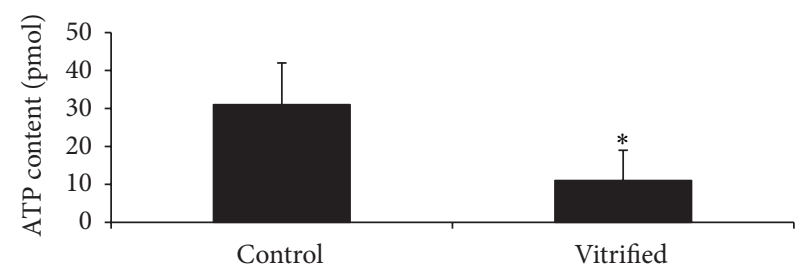

FIGURE 1: ATP content (pmol) of GV oocytes from fresh and vitrified mouse ovaries. ${ }^{*}$ Significant difference between groups $(P<0.05)$.

$50 \mathrm{~W}$ for $1 \mathrm{~min}$, and centrifuged at $10,000 \times \mathrm{g}$ for $20 \mathrm{~min}$ at $4^{\circ} \mathrm{C}$, and the supernatants were collected. Fluorescence was monitored in the supernatant using a spectrofluorometer at $488 \mathrm{~nm}$ excitation and at $525 \mathrm{~nm}$ emission [31]. Data were expressed as $\mu \mathrm{M} \mathrm{H}_{2} \mathrm{O}_{2}$ and the mean dichlorofluorescein (DCF) fluorescence intensity (means \pm SEM). The analysis for each sample was done duplicately.

2.11. Statistical Analysis. All experiments were repeated at least three times. Oocyte maturation and embryo development were analyzed by one-way ANOVA, and Tukey's HSD was used as post hoc tests. ATP levels and RFI values for JC1 and Fluo-4 AM were analyzed statistically by the $t$-test and were considered significant at $P<0.05$.

\section{Results}

3.1. Maturation Rates of Vitrified and Nonvitrified TissueIsolated GV Oocytes. The rates of morphologically normal isolated GV oocytes derived from fresh and vitrified mouse ovaries were $97 \%$ and $89 \%$, respectively. No significant differences were observed in the proportion of normal oocytes between the two groups.

Table 1 summarizes the maturation and fertilization rates of the GV oocytes and their subsequent embryo development. The MII stage was reached by $86.9 \% \pm 4.51$ of GV oocytes in the control group and $50.20 \% \pm 6.97$ in the vitrified group. The rates in the vitrified samples were lower than those in controls $(P<0.05)$.

Fertilization rates were $80.97 \% \pm 3.46$ in the control group and $57.30 \% \pm 10.85$ in the vitrified group, and blastocyst formation rates were $40.98 \pm 2.74$ and $11.36 \% \pm 5.51$ in control and vitrified groups, respectively. The rates in the vitrified samples were lower than those in controls $(P<0.05)$.

3.2. ATP Content of Tissue-Isolated GV Oocytes. As shown in Figure 1, the ATP content of individual GV oocytes derived 


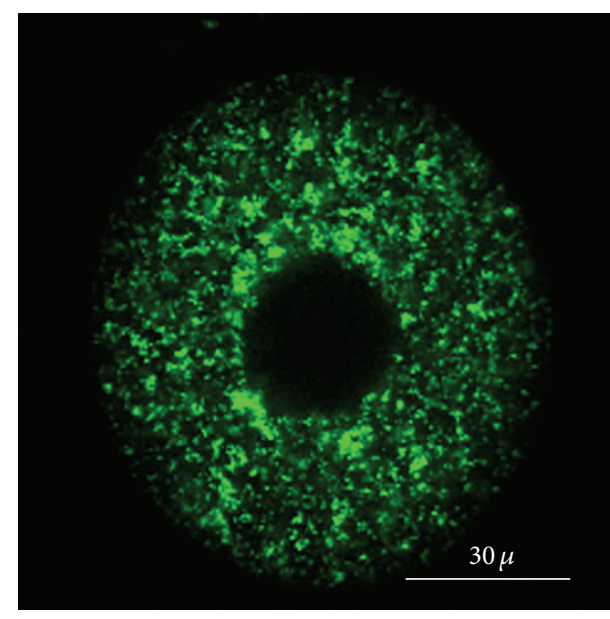

(a)

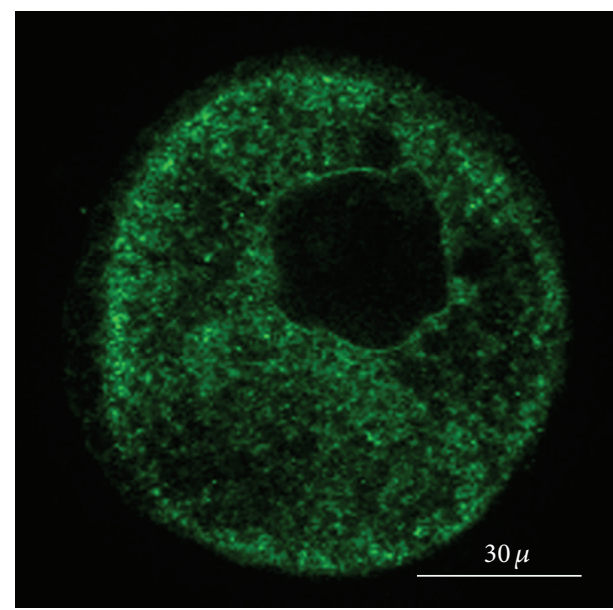

(b)

FIgURE 2: Distribution of viable mitochondria stained by R123. The mitochondrial pattern was (a) uniform and large aggregates or (b) small aggregates.

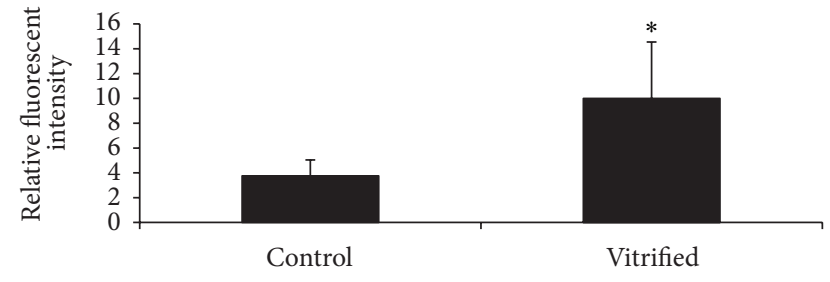

Figure 3: Cytoplasmic free calcium levels measured by relative fluorescence intensity with Flou-4 AM staining in control and vitrified groups of GV oocytes. * Significant difference between groups $(P<0.05)$.

from vitrified samples $\left(11 \times 10^{-12} \pm 8 \times 10^{-12} \mathrm{~mol}\right)$ was significantly lower $(P<0.05)$ than that of fresh samples $(31 \times$ $\left.10^{-12} \pm 11 \times 10^{-12} \mathrm{~mol}\right)$.

3.3. Mitochondrial Distribution. Laser scanning confocal microscopy (LSCM) analysis of GV oocytes loaded with rhodamine 123 (R123) showed two distinct mitochondrial distribution patterns in the ooplasm (Figures 2(a) and 2(b)): uniform, large aggregates and uniform, small aggregates. $66.6 \%$ of the GV oocytes from fresh tissue had large aggregates, and $33.3 \%$ had small aggregates. In the vitrified group, $50 \%$ of the oocytes showed large aggregates, and $50 \%$ showed small ones.

3.4. Intracellular Free Calcium in Tissue-Isolated GV Oocytes. Cytoplasmic free calcium concentrations were quantified using Flou-4 AM staining. The relative fluorescence intensity was $3.753 \pm 1.29$ in fresh samples and $10 \pm 4.55$ in vitrified samples (Figure 3). Differences between the vitrified and fresh groups were significant $(P<0.05)$.

3.5. Mitochondrial Membrane Potential $(\triangle \Psi)$ of TissueIsolated GV Oocytes. To detect mitochondrial membrane potential, GV oocytes were stained with JC-1 monomer.
Oocytes exhibited slightly punctate J-aggregate fluorescence (Figure 4). The red/green ratio of relative fluorescence intensity was $0.484 \pm 0.19$ in fresh samples and $0.47 \pm 0.12$ in vitrified samples (Figure 5), which was not a significant difference.

3.6. ROS Content in GV Oocytes. ROS levels in GV oocytes derived from non-vitrified and vitrified ovarian tissue are shown in Figure 6. The level of ROS in vitrified (1.57 \pm $0.017 \mu \mathrm{mol})$ and non-vitrified $(1.56 \pm 0.020 \mu \mathrm{mol})$ samples was not statistically different.

\section{Discussion}

Vitrification of ovarian tissue is an alternative method for preserving the fertility of cancer patients before they undergo chemotherapy or radiotherapy [32]. Cryopreservation of immature (GV) and MII oocytes, in combination with ovarian tissue vitrification, may be effective for fertility preservation. Vitrification of oocytes at different developmental stages has been successful [33], but direct vitrification of follicles was showed to be better than the vitrification of isolated follicles derived from ovarian tissue [3]. However, data on the developmental rates of immature (GV) oocytes collected from vitrified ovarian tissue is limited, thus, this study has focused on this subject.

We show here that vitrified mouse ovaries yielded a high proportion of morphologically normal GV oocytes; however, their in vitro development and fertilization rates were lower than oocytes from nonvitrified ovaries. Our previous reports and other studies show that the morphology and ultrastructure of mouse oocytes and follicular cells are well preserved after vitrification and subsequent warming of ovarian tissue $[7,26,27,34]$. However, the low fertilization and developmental rates of vitrified GV oocytes could be due to changes that are not visible at light and electron microscopic levels or some changes which need more time to affect the development of oocyte. On the other hand, the vitrified oocytes may be more sensitive than non-vitrified samples to 


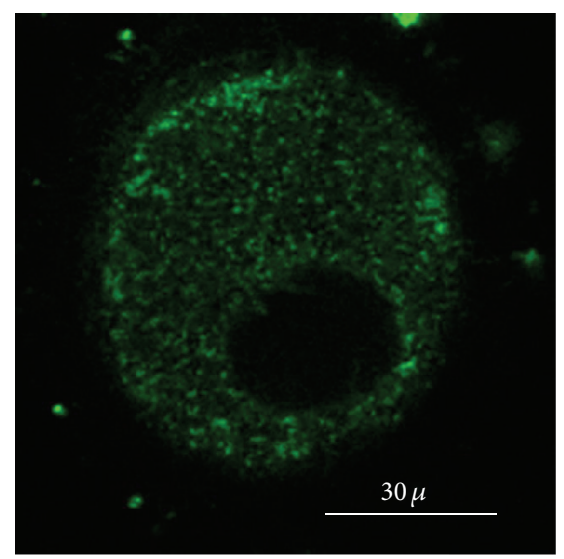

(a)

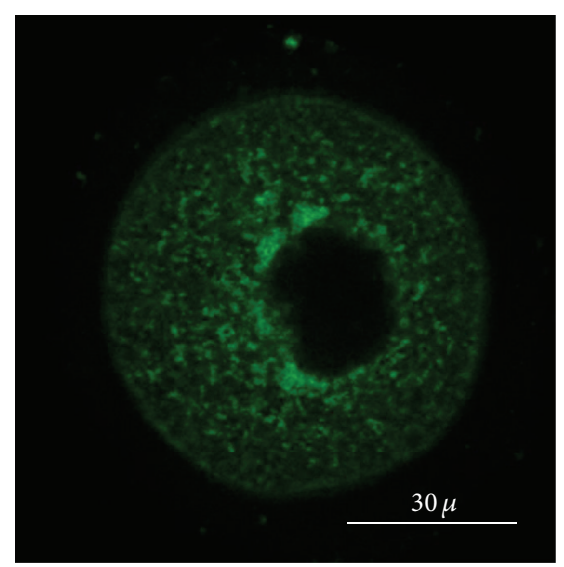

(d)

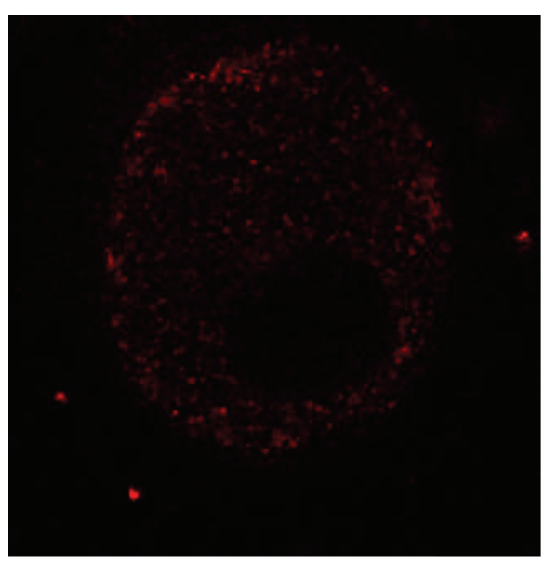

(b)

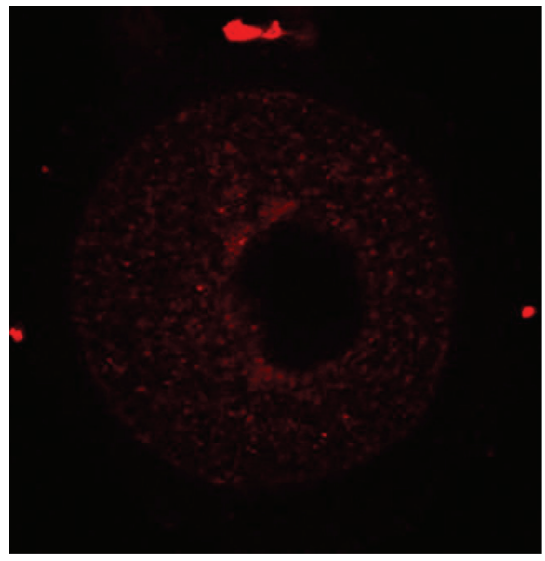

(e)

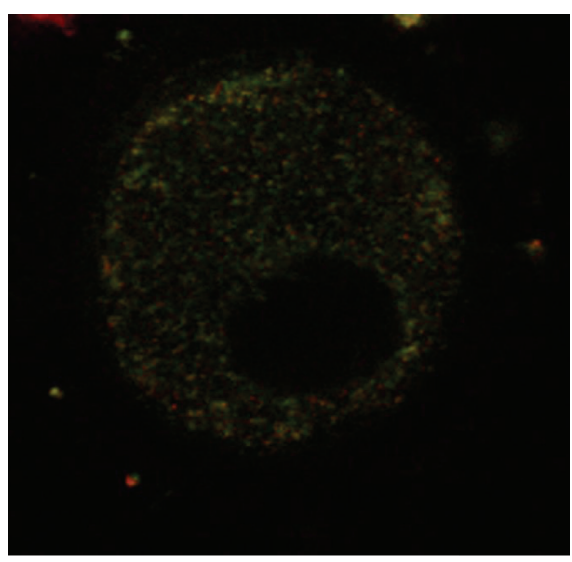

(c)

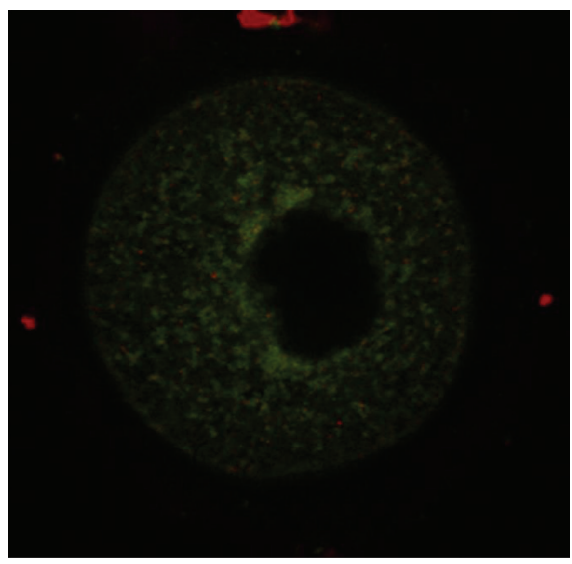

(f)

FIGURE 4: The confocal images of GV oocytes were stained with JC-1. Green and red mitochondria were viewed at 515-530 and 585 nm, respectively. Fresh ((a)-(c)) and vitrified GV oocytes ((d)-(f)): the figures were merged at third column ((c) and (f)). Green (JC-1 monomers) indicating low-polarized mitochondria and red (J-aggregates) denoting high-polarized mitochondria.

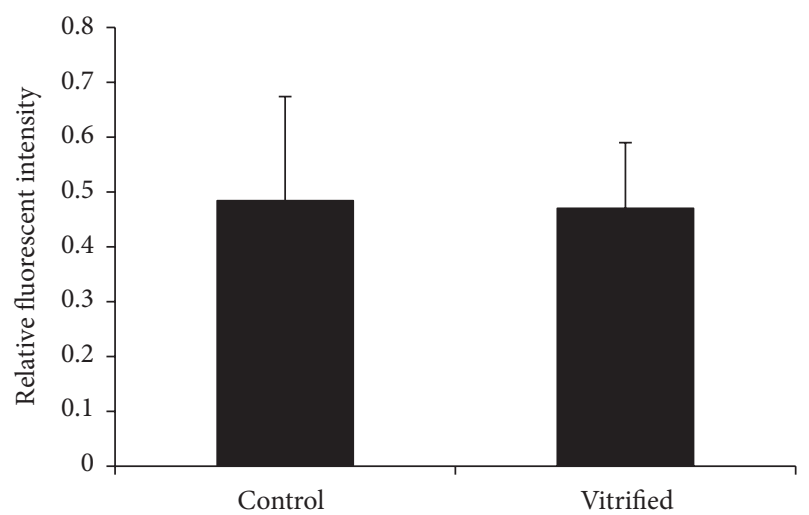

FIGURE 5: Mitochondrial membrane potential calculated using the ratio of relative fluorescence intensity of red to green in JC-1 stained GV oocytes from fresh and vitrified ovaries.

mechanical isolation procedure and/or the damages which were created during the isolation technique which affect their development.

In this study, we focused on oocyte mitochondrial changes after vitrification and warming. Mitochondria are the

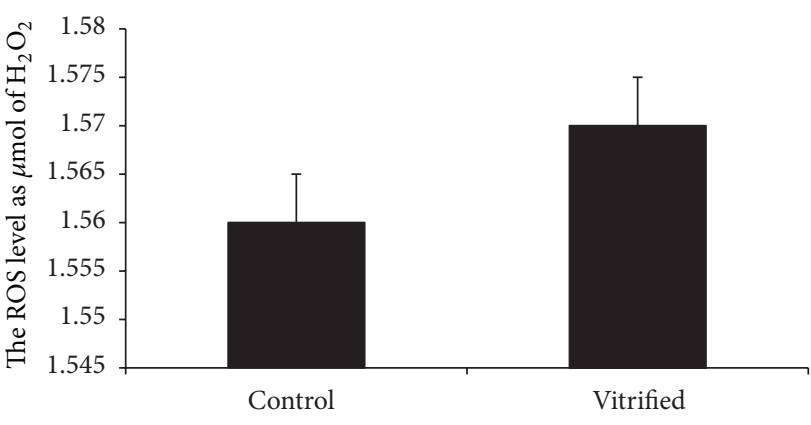

FIGURE 6: Reactive oxygen species (ROS) levels in GV oocytes derived from nonvitrified (control) and vitrified mouse ovaries. There was no significant difference (data as mean $\pm \mathrm{SD}$ ).

critical organelles in the oocyte and contribute to several important cell functions including ATP production. We analyzed mitochondria biochemically to understand some of these changes during the cooling and warming procedure.

Our results demonstrated that ATP concentration was significantly reduced in vitrified samples compared with oocytes from fresh tissue. Inadequate ATP production in 
vitrified GV oocytes may result in delayed and decreased development and maturation of oocytes. Similar results were reported by Gualtieri et al. [35] using slow cooling cryopreservation of human MII oocytes. They demonstrated mitochondrial damage and reduced developmental competence of cryopreserved oocytes.

Specific events during embryo development are ATP dependent including pronucleus formation, syngamy, embryo genome activation, successive cell cleavage, compaction, lineage differentiation, and blastocoel formation [36, 37]. Therefore, a reduction in the ATP content of vitrified GV oocyte could be a reason for impairment of oocyte fertilization and embryo development.

Furthermore, the localization of mitochondria within the ooplasm is important for oocyte development. Mitochondria are mainly localized in regions that have more energy requirements [36, 38-40]. Our LSCM observation showed that the mitochondrial distribution pattern differed between fresh and vitrified groups. The reduction in ATP content of vitrified samples might influence microtubulemediated mitochondrial reorganization. It was suggested that altered mitochondrial distribution affected the developmental potential of vitrified mouse ovary-derived oocytes [41].

An earlier study demonstrated that mouse GV oocytes with abnormal mitochondrial distribution fail to develop to the MII stage [41] and small foci of mitochondria at the cortical part of GV oocytes are characteristic of arrested oocytes $[39,42]$.

Nagai et al. [41] demonstrated mitochondrial dysfunction and fragmentation in mouse MII oocytes after vitrification and warming. They concluded that an abnormal mitochondrial distribution in MII oocytes is one of the causes of developmental retardation and arrest. Vitrification has been suggested to alter important parameters of mitochondria distribution and organization in porcine MII oocytes [43].

Mitochondrial membrane potential is an indication of mitochondrial function [44]. Our LSCM observation using JC-1 staining showed that the $\triangle \Psi$ was similar in both study groups. Hence, ovarian tissue vitrification did not affect the mitochondrial polarity of mouse GV oocytes, but recently Demant et al. demonstrated that mitochondrial inner membrane potential differed significantly between control and vitrified GV oocytes; however, it was similar after 12 days of culture [11].

As we demonstrated that in other part of this study the ATP levels in vitrified samples were lower than the fresh groups, the mitochondrial inner membrane potential was not different in these groups. One explanation for this observation may be due to a reduction in the ATP synthesis within the ooplasm; however, it needs more study.

Mitochondria are important regulators of free intracellular calcium homeostasis. In this study, we showed for the first time that the intracellular free calcium in vitrified $G V$ oocytes increased compared to non-vitrified samples. This could be due to damage to the cell membrane or mitochondria during vitrification. An increase in intracellular calcium during vitrification could be due to influx from outside the cell after damage to the cell membrane or could come from mitochondria or the endoplasmic reticulum, which are intracellular calcium stores [45-48]. Lowther et al. reported that disruption of endoplasmic reticulum during vitrification of mouse oocytes and deficiencies in factors involved in endoplasmic reticulum reorganization during oocyte maturation could contribute to the low development potential of vitrified in vitro-matured oocytes [49].

Successful fertilization and embryo cleavage are both ATP and calcium dependent. Changes in their levels could affect not only fertilization rate, but also embryonic development [49-51]. An increase in oocyte calcium during vitrification could be involved in premature exocytosis of cortical granules resulting in zona pellucida hardening that prevents sperm penetration. A similar observation was reported after exposure of oocytes to cryoprotectant solution [52, 53].

However, in other part of this study, our result showed that there was no significant increase in the ROS level of vitrified samples in comparison with their control. It means that, in spitve of some changes in the mitochondria of vitrified $\mathrm{GV}$, these alterations could not cause an increase in oxidative stress. Furthermore, the high level of ROS could induce apoptotic cell death by mitochondrial apoptotic pathways [54].

However, this study was focused on the GV oocytes and more studies need to compare the effects of this cryopreservation technique on the MII stage oocyte.

\section{Conclusion}

In conclusion, vitrification of mouse ovaries using the vitrification medium EFS40 changed the mitochondrial distribution, reduced the ATP content, and increased intracellular free calcium in isolated ovarian GV oocytes. However, vitrification had no effect on mitochondrial membrane polarity. These changes could cause delays in maturation and impede the development and survival of GV oocytes.

\section{Acknowledgments}

The authors thank Mrs. Shabnam Abdi for ROS analysis technical assistant. This work was supported by Iran National Science Foundation (Mojdeh Salehnia) and the Karolinska Institute Foundation (Jose Inzunza and Virpi Töhönen).

\section{References}

[1] K. Mochida, A. Hasegawa, M. W. Li et al., "High osmolality vitrification: a new method for the simple and temperaturepermissive cryopreservation of mouse embryos," PLoS ONE, vol. 8, no. 1, Article ID e49316, 2013.

[2] N. Desai, F. AbdelHafez, M. Y. Ali et al., "Mouse ovarian follicle cryopreservation using vitrification or slow programmed cooling: assessment of in vitro development, maturation, ultrastructure and meiotic spindle organization," Journal of Obstetrics and Gynaecology Research, vol. 37, no. 1, pp. 1-12, 2011.

[3] W.-J. Choi, H.-J. Yeo, J.-K. Shin, S.-A. Lee, J.-H. Lee, and W.-Y. Paik, "Effect of vitrification method on survivability, follicular growth and ovulation of preantral follicles in mice," Journal of Obstetrics and Gynaecology Research, vol. 33, no. 2, pp. 128-133, 2007. 
[4] S. K. Cha, B. Y. Kim, M. K. Kim et al., "Effects of various combinations of cryoprotectants and cooling speed on the survival and further development of mouse oocytes after vitrification," Clinical Experimental Reproductive Medicin, vol. 38, no. 1, pp. 24-30, 2011.

[5] D. Boonkusol, T. Faisaikarm, A. Dinnyes, and Y. Kitiyanant, "Effects of vitrification procedures on subsequent development and ultrastructure of in vitro-matured swamp buffalo (Bubalus bubalis) oocytes," Reproduction, Fertility and Development, vol. 19, no. 2, pp. 383-391, 2007.

[6] K. Haidari, M. Salehnia, and M. Rezazadeh Valojerdi, "The effect of leukemia inhibitory factor and coculture on the in vitro maturation and ultrastructure of vitrified and nonvitrified isolated mouse preantral follicles," Fertility and Sterility, vol. 90, no. 6, pp. 2389-2397, 2008.

[7] A. Abedelahi, M. Salehnia, A. A. Allameh, and D. Davoodi, "Sodium selenite improves the in vitro follicular development by reducing the reactive oxygen species level and increasing the total antioxidant capacity and glutathione peroxide activity," Human Reproduction, vol. 25, no. 4, pp. 977-985, 2010.

[8] M. Segino, M. Ikeda, F. Hirahara, and K. Sato, "In vitro follicular development of cryopreserved mouse ovarian tissue," Reproduction, vol. 130, no. 2, pp. 187-192, 2005.

[9] A. Jones, J. Van Blerkom, P. Davis, and A. A. Toledo, "Cryopreservation of metaphase II human oocytes effects mitochondrial membrane potential: implications for developmental competence," Human Reproduction, vol. 19, no. 8, pp. 1861-1866, 2004.

[10] Y. Liang, F.-Y. Ning, W.-J. Du, C.-S. Wang, S.-H. Piao, and T.-Z. An, "The type and extent of injuries in vitrified mouse oocytes," Cryobiology, vol. 64, no. 2, pp. 97-102, 2012.

[11] M. Demant, T. Trapphoff, T. Fröhlich, G. J. Arnold, and U. Eichenlaub-Ritter, "Vitrification at the pre-antral stage transiently alters inner mitochondrial membrane potential but proteome of in vitro grown and matured mouse oocytes appears unaffected," Human Reproduction, vol. 27, no. 4, pp. 1096-1111, 2012.

[12] C.-L. Yan, X.-W. Fu, G.-B. Zhou, X.-M. Zhao, L. Suo, and S.E. Zhu, "Mitochondrial behaviors in the vitrified mouse oocyte and its parthenogenetic embryo: effect of Taxol pretreatment and relationship to competence," Fertility and Sterility, vol. 93, no. 3, pp. 959-966, 2010.

[13] X.-M. Zhao, W.-H. Du, D. Wang et al., "Effect of cyclosporine pretreatment on mitochondrial function in vitrified bovine mature oocytes," Fertility and Sterility, vol. 95, no. 8, pp. 27862788, 2011.

[14] X.-M. Zhao, W.-H. Du, D. Wang et al., "Recovery of mitochondrial function and endogenous antioxidant systems in vitrified bovine oocytes during extended in vitro culture," Molecular Reproduction and Development, vol. 78, no. 12, pp. 942-950, 2011.

[15] X.-M. Zhao, X.-W. Fu, Y.-P. Hou et al., "Effect of vitrification on mitochondrial distribution and membrane potential in mouse two pronuclear (2-PN) embryos," Molecular Reproduction and Development, vol. 76, no. 11, pp. 1056-1063, 2009.

[16] M. D. L. Reyes, J. Palomino, V. H. Parraguez, M. Hidalgo, and P. Saffie, "Mitochondrial distribution and meiotic progression in canine oocytes during in vivo and in vitro maturation," Theriogenology, vol. 75, no. 2, pp. 346-353, 2011.

[17] S. H. El Shourbagy, E. C. Spikings, M. Freitas, and J. C. St. John, "Mitochondria directly influence fertilisation outcome in the pig," Reproduction, vol. 131, no. 2, pp. 233-245, 2006.
[18] S. Liu, Y. Li, X. Gao, J.-H. Yan, and Z.-J. Chen, "Changes in the distribution of mitochondria before and after in vitro maturation of human oocytes and the effect of in vitro maturation on mitochondria distribution," Fertility and Sterility, vol. 93, no. 5, pp. 1550-1555, 2010.

[19] Q. Y. Sun, G. M. Wu, L. Lai et al., "Translocation of active mitochondria during pig oocyte maturation, fertilization and early embryo development in vitro," Reproduction, vol. 122, no. 1, pp. 155-163, 2001.

[20] L.-Y. Wang, D.-H. Wang, X.-Y. Zou, and C.-M. Xu, "Mitochondrial functions on oocytes and preimplantation embryos," Journal of Zhejiang University B, vol. 10, no. 7, pp. 483-492, 2009.

[21] J. Müller-Höcker, S. Schäfer, S. Weis, C. Münscher, and T. Strowitzki, "Morphological-cytochemical and molecular genetic analyses of mitochondria in isolated human oocytes in the reproductive age," Molecular Human Reproduction, vol. 2, no. 12, pp. 951-958, 1996.

[22] P. Reynier, P. May-Panloup, M.-F. Chrétien et al., "Mitochondrial DNA content affects the fertilizability of human oocytes," Molecular Human Reproduction, vol. 7, no. 5, pp. 425-429, 2001.

[23] T. A. Santos, S. El Shourbagy, and J. C. St. John, "Mitochondrial content reflects oocyte variability and fertilization outcome," Fertility and Sterility, vol. 85, no. 3, pp. 584-591, 2006.

[24] J. Van Blerkom, P. Davis, and S. Alexander, "Differential mitochondrial distribution in human pronuclear embryos leads to disproportionate inheritance between blastomeres: relationship to microtubular organization, ATP content and competence," Human Reproduction, vol. 15, no. 12, pp. 2621-2633, 2000.

[25] S. Manipalviratn, Z.-B. Tong, B. Stegmann, E. Widra, J. Carter, and A. Decherney, "Effect of vitrification and thawing on human oocyte ATP concentration," Fertility and Sterility, vol. 95, no. 5, pp. 1839-1841, 2011.

[26] M. Salehnia, E. A. Moghadam, and M. R. Velojerdi, "Ultrastructure of follicles after vitrification of mouse ovarian tissue," Fertility and Sterility, vol. 78, no. 3, pp. 644-645, 2002.

[27] M. R. Valojerdi and M. Salehnia, "Developmental potential and ultrastructural injuries of metaphase II (MII) mouse oocytes after slow freezing or vitrification," Journal of Assisted Reproduction and Genetics, vol. 22, no. 3, pp. 119-127, 2005.

[28] J. Van Blerkom, P. W. Davis, and J. Lee, "ATP content of human oocytes and developmental potential and outcome after in-vitro fertilization and embryo transfer," Human Reproduction, vol. 10, no. 2, pp. 415-424, 1995.

[29] J. Van Blerkom, P. Davis, and S. Alexander, "Inner mitochondrial membrane potential $(\Delta \Psi \mathrm{m})$, cytoplasmic ATP content and free $\mathrm{Ca}^{2+}$ levels in metaphase II mouse oocytes," Human Reproduction, vol. 18, no. 11, pp. 2429-2440, 2003.

[30] S. T. Smiley, M. Reers, C. Mottola-Hartshorn et al., "Intracellular heterogeneity in mitochondrial membrane potentials revealed by a J-aggregate-forming lipophilic cation JC-1," Proceedings of the National Academy of Sciences of the United States of America, vol. 88, no. 9, pp. 3671-3675, 1991.

[31] C. P. LeBel, H. Ischiropoulos, and S. C. Bondy, "Evaluation of the probe $2 /, 7 I$-dichlorofluorescin as an indicator of reactive oxygen species formation and oxidative stress," Chemical Research in Toxicology, vol. 5, no. 2, pp. 227-231, 1992.

[32] H. J. Chang, J. H. Moon, J. R. Lee, B. C. Jee, C. S. Suh, and S. H. Kim, "Optimal condition of vitrification method for cryopreservation of human ovarian cortical tissues," Journal of Obstetrics and Gynaecology Research, vol. 37, no. 8, pp. 10921101, 2011. 
[33] J. Saragusty and A. Arav, "Current progress in oocyte and embryo cryopreservation by slow freezing and vitrification," Reproduction, vol. 141, no. 1, pp. 1-19, 2011.

[34] T. Mazoochi, M. Salehnia, M. R. Valojerdi, and S. J. Mowla, "Morphologic, ultrastructural, and biochemical identification of apoptosis in vitrified-warmed mouse ovarian tissue," Fertility and Sterility, vol. 90, no. 4, pp. 1480-1486, 2008.

[35] R. Gualtieri, M. Iaccarino, V. Mollo, M. Prisco, S. Iaccarino, and R. Talevi, "Slow cooling of human oocytes: ultrastructural injuries and apoptotic status," Fertility and Sterility, vol. 91, no. 4, pp. 1023-1034, 2009.

[36] J. Ramalho-Santos, S. Varum, S. Amaral, P. C. Mota, A. P. Sousa, and A. Amaral, "Mitochondrial functionality in reproduction: from gonads and gametes to embryos and embryonic stem cells," Human Reproduction Update, vol. 15, no. 5, pp. 553-572, 2009.

[37] A. M. Tarazona, J. I. Rodríguez, L. F. Restrepo, and M. OliveraAngel, "Mitochondrial activity, distribution and segregation in bovine oocytes and in embryos produced in vitro," Reproduction in Domestic Animals, vol. 41, no. 1, pp. 5-11, 2006.

[38] B. D. Bavister and J. M. Squirrell, "Mitochondrial distribution and function in oocytes and early embryos," Human Reproduction, vol. 15, no. 2, pp. 189-198, 2000.

[39] P. G. Calarco, "Polarization of mitochondria in the unfertilized mouse oocyte," Developmental Genetics, vol. 16, no. 1, pp. 36-43, 1995.

[40] J. Van Blerkom, P. Davis, and S. Alexander, "Differential mitochondrial distribution in human pronuclear embryos leads to disproportionate inheritance between blastomeres: relationship to microtubular organization, ATP content and competence," Human Reproduction, vol. 15, no. 12, pp. 2621-2633, 2000.

[41] S. Nagai, T. Mabuchi, S. Hirata et al., "Correlation of abnormal mitochondrial distribution in mouse oocytes with reduced developmental competence," Tohoku Journal of Experimental Medicine, vol. 210, no. 2, pp. 137-144, 2006.

[42] W.-Q. Shi, S.-E. Zhu, D. Zhang et al., "Improvement development by Taxol pretreatment after vitrification of in vitro matured porcine oocytes," Reproduction, vol. 131, no. 4, pp. 795804, 2006.

[43] L.-Y. Shi, H.-F. Jin, J.-G. Kim et al., "Ultra-structural changes and developmental potential of porcine oocytes following vitrification," Animal Reproduction Science, vol. 100, no. 1-2, pp. 128-140, 2007.

[44] L. M. Mehlmann, M. Terasaki, L. A. Jaffe, and D. Kline, "Reorganization of the endoplasmic reticulum during meiotic maturation of the mouse oocyte," Developmental Biology, vol. 170, no. 2, pp. 607-615, 1995.

[45] J. Van Blerkom and M. N. Runner, "Mitochondrial reorganization during resumption of arrested meiosis in the mouse oocyte," American Journal of Anatomy, vol. 171, no. 3, pp. 335355, 1984.

[46] M. Reers, S. T. Smiley, C. Mottola-Hartshorn, A. Chen, M. Lin, and L. B. C. Lan Bo Chen, "Mitochondrial membrane potential monitored by JC-1 dye," Methods in Enzymology, vol. 260, pp. 406-417, 1995.

[47] T. Ducibella, R. M. Schultz, and J.-P. Ozil, "Role of calcium signals in early development," Seminars in Cell and Developmental Biology, vol. 17, no. 2, pp. 324-332, 2006.

[48] D. Kline, L. Mehlmann, C. Fox, and M. Terasaki, “The cortical endoplasmic reticulum (ER) of the mouse egg: localization of ER clusters in relation to the generation of repetitive calcium waves," Developmental Biology, vol. 215, no. 2, pp. 431-442, 1999.
[49] K. M. Lowther, V. N. Weitzman, D. Maier, and L. M. Mehlmann, "Maturation, fertilization, and the structure and function of the endoplasmic reticulum in cryopreserved mouse oocytes," Biology of Reproduction, vol. 81, no. 1, pp. 147-154, 2009.

[50] T. Ducibella and R. Fissore, "The roles of $\mathrm{Ca}^{2+}$, downstream protein kinases, and oscillatory signaling in regulating fertilization and the activation of development," Developmental Biology, vol. 315, no. 2, pp. 257-279, 2008.

[51] C. Gómez-Fernández, A. M. López-Guerrero, E. Pozo-Guisado, I. S. Álvarez, and F. J. Martín-Romero, "Calcium signaling in mouse oocyte maturation: the roles of STIM1, ORAI1 and SOCE," Molecular Human Reproduction, vol. 18, no. 4, pp. 194203, 2012.

[52] S. Miyazaki, "Thirty years of calcium signals at fertilization," Seminars in Cell and Developmental Biology, vol. 17, no. 2, pp. 233-243, 2006.

[53] M. G. Larman, C. B. Sheehan, and D. K. Gardner, "Calcium-free vitrification reduces cryoprotectant-induced zona pellucida hardening and increases fertilization rates in mouse oocytes," Reproduction, vol. 131, no. 1, pp. 53-61, 2006.

[54] J. Chandra, A. Samali, and S. Orrenius, "Triggering and modulation of apoptosis by oxidative stress," Free Radical Biology and Medicine, vol. 29, no. 3-4, pp. 323-333, 2000. 


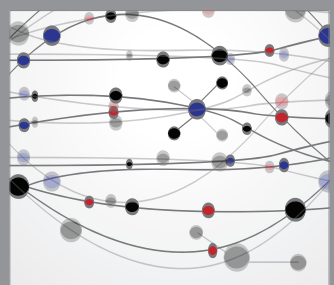

The Scientific World Journal
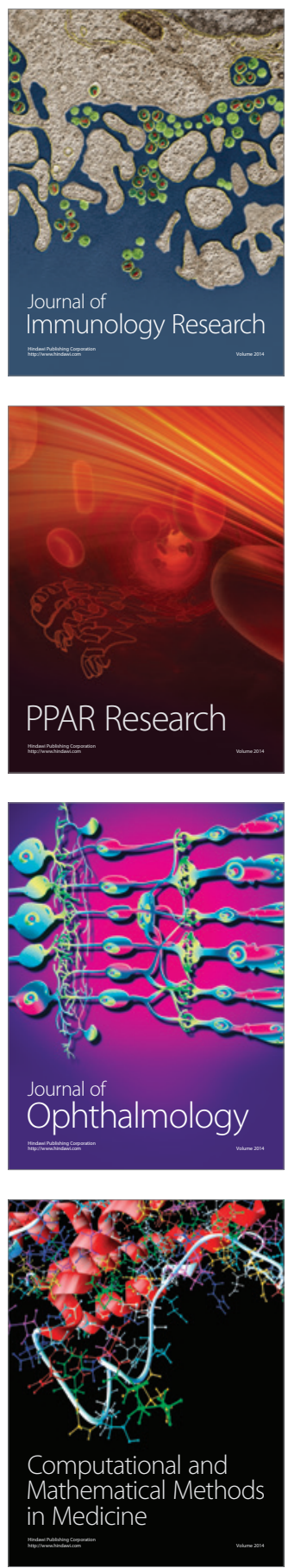

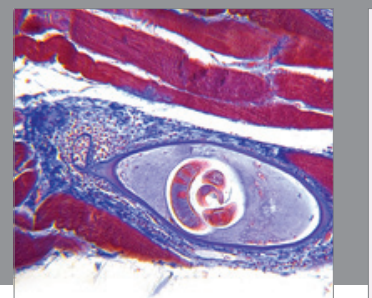

Gastroenterology

Research and Practice
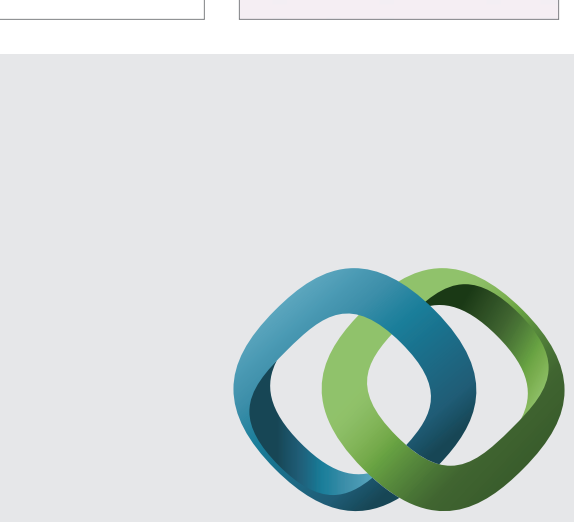

\section{Hindawi}

Submit your manuscripts at

http://www.hindawi.com
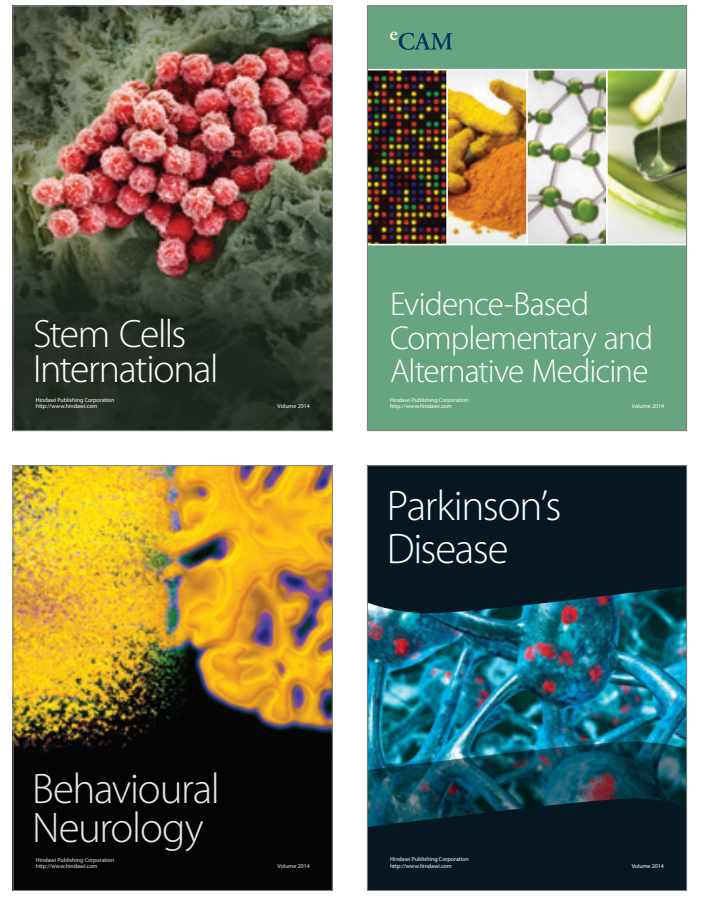
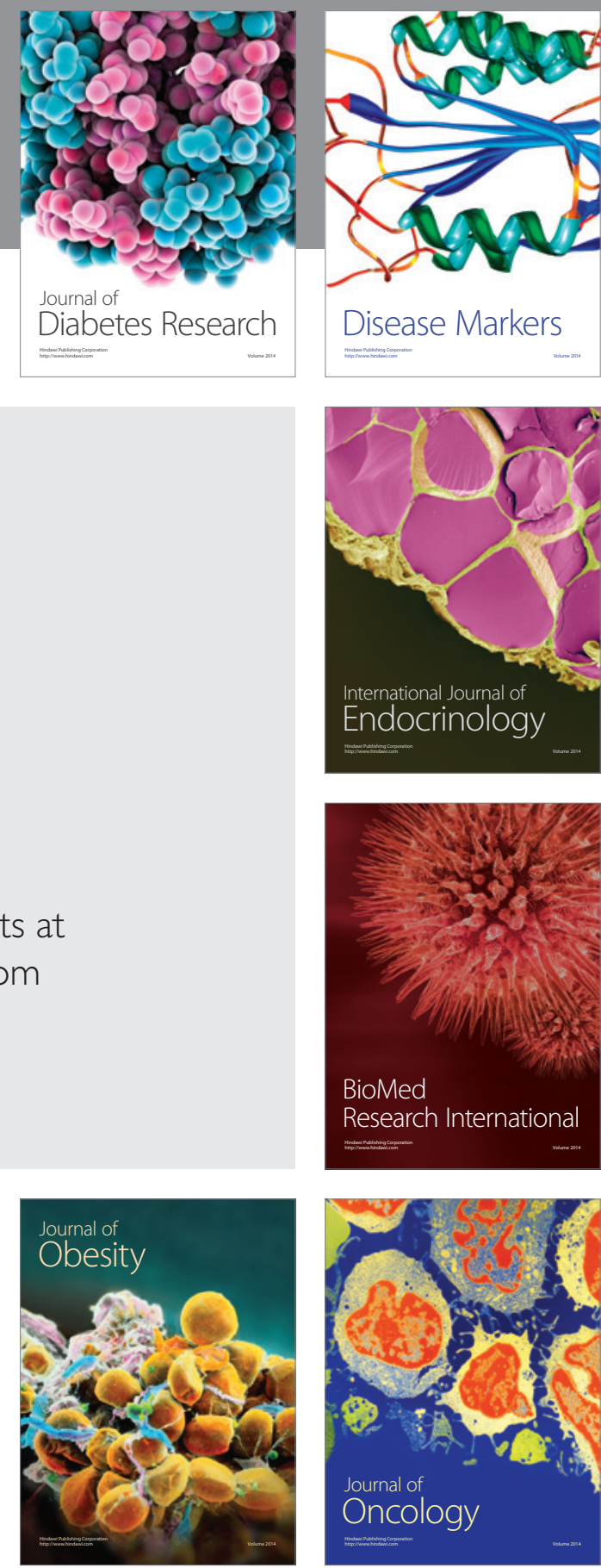

Disease Markers
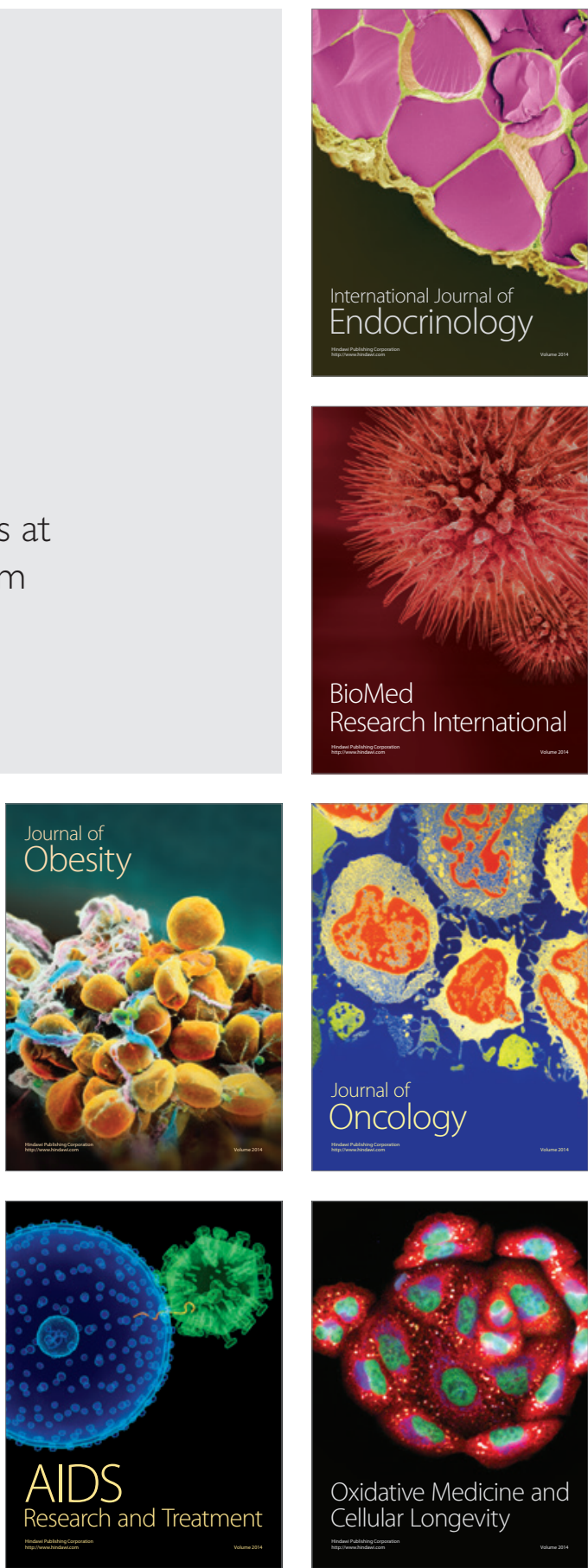